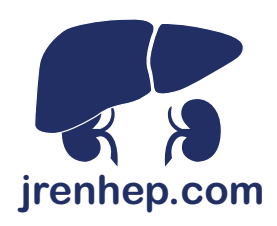

CASE REPORT

\title{
Plasmapheresis in the Management of Acute Pancreatitis due to Severe Hypertriglyceridemia-Reporting New Cases
}

\author{
Andreia Campos, Josefina Santos, Cristina Freitas, Ana Castro, Sofia Santos, João Pedro Pimentel, \\ António Cabrita
}

Nephrology Department, Centro Hospitalar do Porto, Porto, Portugal

\begin{abstract}
Acute pancreatitis is a potentially life-threatening disease. If the diagnosis and the treatment are not prompt, it can rapidly evolve to a medical emergency. Severe hypertriglyceridemia, defined as above $1000 \mathrm{mg} / \mathrm{dl}$, is the third most common cause of acute pancreatitis. Conventional management includes fat dietary restriction and pharmacological treatment; however, these measures take time to be effective. Plasmapheresis seems to be an alternative and safe adjunctive therapy because it allows the rapid reduction of the trigger agent in circulation. Its use, especially in severe cases, has been increasingly reported. The authors report three cases of severe hypertriglyceridemia-induced pancreatitis in which early plasmapheresis was successfully used with other supportive clinical management.
\end{abstract}

Keywords: acute pancreatitis; plasmapheresis; severe hypertriglyceridemia

Received: 24 November 2016; Accepted after revision: 21 December 2016; Published: 03 February 2017.

Author for correspondence: Andreia Campos, Serviço de Nefrologia (Nephrology Department), Centro Hospitalar do Porto, Largo do Prof. Abel Salazar, 4099-001 Porto, Portugal. Email: andcriscampos@hotmail.com

How to cite: Campos A et al. Plasmapheresis in the management of acute pancreatitis due to severe hypertriglyceridemia-reporting new cases. J Ren Hepat Disord 2017;1(1):29-34.

DOI: http://dx.doi.org/10.15586/jrenhep.2017.3

Copyright: Campos A et al.

License: This open access article is licensed under Creative Commons Attribution 4.0 International (CC BY 4.0).

http://creativecommons.org/licenses/by/4.0

\section{Introduction}

Acute pancreatitis (AP) is a potentially life-threatening disease. Without prompt diagnosis and treatment, it can rapidly evolve to a medical emergency, leading into pancreatic necrosis and ultimately to death $(1,2)$. Serum hypertriglyceridemia (HTG) is the third most frequent cause of AP after gallstones and alcohol, with a reported incidence of $1 \%-10 \%$ (3). Serum triglyceride levels $>1000 \mathrm{mg} / \mathrm{dl}$, also known as severe hypertriglyceridemia (SHTG), are usually needed to trigger AP (4-6).

Conventional management of SHTG includes fat dietary restriction and pharmacological treatment; however, the slow action of antihyperlipidemic therapy poses a problem when the situation requires a rapid reduction of the offending agent in circulation (2). Plasmapheresis (PF) seems to be an alternative and safe therapy for these kinds of cases because it allows the rapid reduction of triglycerides (TG) in just a few hours $(7,8)$. Its use, especially in high-risk patients admitted in intensive care units, has been increasingly reported. Unfortunately, there are no controlled or randomized studies to prove its benefit in mortality reduction. Most of the data are based on single case reports. That is why the American Society for Apheresis (ASFA) recommendation for this indication is only Grade 2C (9). PF does not interfere in TG production, and therefore, it should always be considered as an adjunctive therapy to medical treatment. 
In our hospital, $\mathrm{PF}$ is a technique performed by nephrologists, regardless of whether the indication is nephrologic or not.

Due to the importance of describing all new cases, the authors report three cases of SHTG-induced AP in which early PF was used with other supportive clinical management.

\section{Case Reports}

The patients were admitted to the emergency department with an AP; the etiology factor identified was SHTG. All of them had an unfavorable course under medical management in the first $24-48 \mathrm{~h}$. There was no previous available lipid profile, history of treatment with cholesterol or TG-lowering drugs, or history of gallstones or relevant family history. PF was done according to TG levels. A temporary central venous catheter was placed with no complications. A double membrane filtration device and $5 \%$ albumin as the replacement fluid were used. The volume treated was correspondent to one plasma volume and the duration of the session was according to the replacement. Nonfractioned heparin was used for anticoagulation. There were no PF-related complications. At discharge, the patients were medicated with lower lipid drugs. At follow-up, as an outpatient, all of them were asymptomatic and their TG and pancreatic enzyme levels were within the normal range.

To clarify, data in Table 1 refer to variables at admission. In Figure 1, it is represented as the effect of PF on serum TG.

\section{Case 1}

This is a case of a 43-year-old male with type 2 diabetes diagnosed 6 years before, with poor metabolic control, dyslipidemia, and obesity (body mass index (BMI): $30.6 \mathrm{~kg} / \mathrm{m}^{2}$ ). $\mathrm{He}$ had no history of gallstones or alcohol intake. He was admitted with an epigastric abdominal pain with dorsal irradiation, nausea, and vomiting that begun $24 \mathrm{~h}$ before. On examination, he had no fever, pulse rate was $124 / \mathrm{min}$, and blood pressure was 100/60 $\mathrm{mm} \mathrm{Hg}$. He was prostrate and with signs of dehydration. The abdomen was diffusely

Table 1. Initial laboratory data of patients prior to the plasmapheresis treatment

\begin{tabular}{|c|c|c|c|c|}
\hline Laboratory findings & Case 1 & Case 2 & Case 3 & Reference values \\
\hline Hemoglobin (g/dl) & 16 & 17 & 16.6 & $13-18$ \\
\hline Hematocrit $(\%)$ & 40 & 38 & 42 & $40-52$ \\
\hline Platelets $/ \mathrm{mm}^{3}$ & 287,000 & 151,000 & 201,000 & $150-440$ \\
\hline Total leucocyte count $/ \mu \mathrm{L}$ & 17670 & 14290 & 5850 & $3800-10600$ \\
\hline CRP (mg/dl) & 327.65 & 56.5 & 337 & $<5.0$ \\
\hline Amylase (U/L) & 663 & 1033 & 70 & $<3.0$ \\
\hline Lipase (U/L) & 2686 & 750 & 2485 & $13-60$ \\
\hline Triglycerides (mg/dl) & 1229 & 5565 & 1348 & $<200$ \\
\hline Total cholesterol (mg/dl) & 630 & 750 & 350 & $<200$ \\
\hline AST/ALT (mg/dl) & $50 / 26$ & $109 / 72$ & $67 / 91$ & $4-50 / 4-33$ \\
\hline Total bilirubin/direct bilirubin (mg/dl) & $0.7 / 0.1$ & $0.9 / 0.2$ & $1.0 / 0.1$ & $0.1-1.1 ; 0.1-0.3$ \\
\hline Sodium (mmol/L) & 122 & 128 & 135 & $135-145$ \\
\hline Calcium (mg/dl) & 9.0 & 8.7 & 8.8 & $8.8-10.2$ \\
\hline Glucose (mg/dl) & 575 & $>400$ & 180 & $60-100$ \\
\hline Lactate desidrogenase (U/L) & 474 & 370 & 259 & $135-225$ \\
\hline Creatinine (mg/dl) & 1.96 & 0.71 & 1.7 & $0.67-1.17$ \\
\hline Urea (mg/dl) & 65 & 28 & 29 & $15-45$ \\
\hline Bicarbonate (mmol/L) & 12 & 16 & 17 & $22-26$ \\
\hline Lactates & 5.0 & 2 & 2.6 & \\
\hline $\mathrm{HbA} 1 \mathrm{C}(\%)$ & 11.6 & 12 & 11 & $<7.0$ \\
\hline
\end{tabular}

AST, aspartate transaminase/ALT, alanine transaminase; CRP, C-reactive protein. 


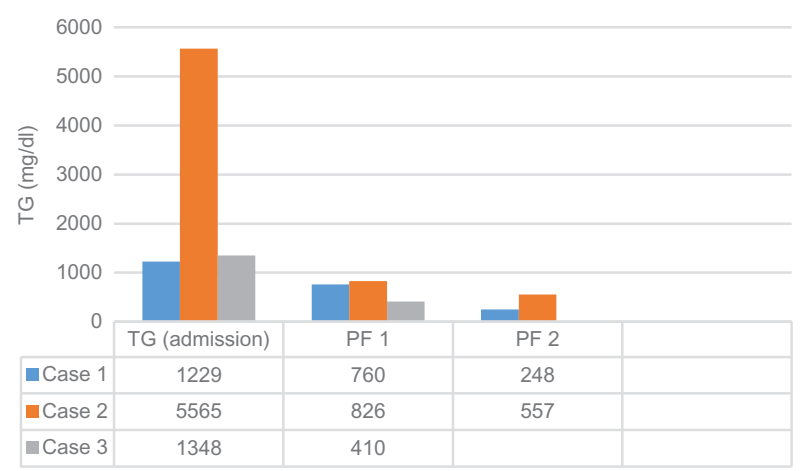

Figure 1. Effect of plasmapheresis on serum triglycerides (TG). PF1: TG after first plasmapheresis treatment; PF2: TG after second plasmapheresis treatment.

painful. He was oliguric. There were no other abnormalities. Laboratory tests showed leukocytosis and neutrophilia, an elevated C-reactive protein (CRP), a diabetic ketoacidosis, hyperlactacidemia; pancreatic alpha-amylase $663 \mathrm{mg} / \mathrm{dl}$, lipase 2686 mg/dl; and TG 1229 (Table 1). Abdominal computed tomography (CT) showed a gallbladder without cholecystitis or lithiasis, pancreas with necrosis $(\sim 90 \%)$, and a necro-hemorrhagic collection in the tail. Delayed renal excretion of iodinated contrast was observed. Microbiological cultures were negative. The patient started on a fasting regimen, and treatment began with endovenous fluids and insulin. The initial response was unfavorable, with sustained hemodynamic instability, respiratory distress, and oliguria. $\mathrm{He}$ was admitted to the intensive care unit with Acute Physiology and Chronic Health Evaluation II (APACHE II) score 14 with need of hemodynamic and respiratory support. TG and lipase increased in the first $24 \mathrm{~h}$ and renal function worsened (creatinine $4.96 \mathrm{mg} / \mathrm{dl}$ ). He was submitted to an urgent $\mathrm{PF}$ on day 2 of admission. The TG decreased to $<1000 \mathrm{mg} / \mathrm{dl}$ and $248 \mathrm{mg} / \mathrm{dl}$ after the first and second sessions of PF, respectively; the pancreatic enzymes normalized. The patient showed significant clinical improvement with resolution of multiorgan dysfunction, namely, renal dysfunction. He was started on oral fat-free diet and fenofibrate on day 4 and discharged from the intensive care unit to the surgery department on day 10. A CT follow-up at $72 \mathrm{~h}$ showed a regression of pancreas necrosis and of hemorrhagic collection, with no signs of infection. A revaluation CT scan was done 2 weeks after showing a complete recovery of pancreatic necrosis. Fortunately, he did not present any late complications of this severe pancreatitis, including acute fluid collections, pancreatic necrosis pseudocysts, abscesses, ascitis and pancreatic fistulas, splenic vein thrombosis, or false aneurysms. On follow-up after 2 months, his TG levels were found normal.

\section{Case 2}

This is a case of a 42-year-old man with type 2 diabetes, hypertension, obesity (BMI $41.5 \mathrm{~kg} / \mathrm{m}^{2}$ ), and a past history of dyslipidemia. He had a schizophrenia and the therapeutic compliance was very poor. He was admitted with a diffuse abdominal pain. On physical examination at admission, he had no fever and was hemodynamically stable. The abdominal examination showed tenderness in the right upper quadrant, with no other abnormalities. Respiratory, cardiovascular, and neurologic examinations were unremarkable.

Laboratory studies at admission showed leukocytosis with neutrophilia, an elevated CRP, and a severe hypertriglyceridemia (TG $5565 \mathrm{mg} / \mathrm{dl}$ ). Other relevant laboratory results at admission and in posterior workup are presented in Table 1. The abdominal CT showed an acute nonlithiasic edematous pancreatitis.

The patient was admitted to the intermediate care unit with APACHE II score 10, and started on a fasting regimen, insulin perfusion, and fluids. In the context of severe pancreatitis and vigorous fluid therapy, he developed signs of heart failure in the first $48 \mathrm{~h}$, requiring oxygen and diuretic therapy. Transthoracic echocardiography did not show any abnormalities. The abdominal pain and the pancreatic markers worsened. In the absence of response to medical therapy during the first $48 \mathrm{~h}$, he started on PF treatment. He needed two consecutive sessions. The procedure was complicated by the clotting of the filters due to the high plasma viscosity requiring high doses of unfractionated heparin. After the first session, his TG levels were $826 \mathrm{mg} / \mathrm{dl}$. One additional PF session was performed in the subsequent day (Figure 1). A complete clinical and analytical recovery was seen on the patient.

\section{Case 3}

A 38-year-old male with uncontrolled type 2 diabetes and a known history of high alcohol consumption (>200 g/day) was admitted to the emergency room with an epigastric pain and nausea. On examination, he was hemodynamically stable. Respiratory and cardiovascular examinations were normal. He presented with abdominal pain and distention. Laboratory tests at admission are shown in Table 1. The abdominal CT showed aspects of AP without dilation of the bile ducts or gallstones.

The blood was highly lipemic and on ultracentrifuge showed TG $1348 \mathrm{mg} / \mathrm{dl}$. The patient was admitted to the intermediate care unit, with APACHE II score 8 . He was initially managed with fasting, glucose control, and enteral fibrate. Despite the medical management, $24 \mathrm{~h}$ after admission, there was a lack of improvement; the renal function worsened with serum creatinine/urea $(2.5 / 85 \mathrm{mg} / \mathrm{dl})$. Therefore, we started PF. He underwent two sessions in consecutive days with clinical, analytical, and imagological improvements.

The patient was discharged 7 days after admission with oral atorvastatin, fenofibrate, and insulin.

\section{Discussion and Conclusions}

AP could be a medical emergency associated with higher morbidity and mortality. Due to potential associated 
complications, such as sepsis, pancreatic necrosis, abscess formation and renal insufficiency, or multiorgan dysfunction, AP is considered a potential life-threatening illness with a mortality rate of up to $7 \%-0 \%(2,8)$. The diagnostic criteria of AP require two of the following three features: characteristic abdominal pain; serum amylase or lipase more than three times the normal upper limit; findings of AP on CT scan $(10,11)$. Our patients presented with all of these criteria. However, in the setting of SHTG, amylase and lipase serum levels may be normal or only mildly raised obscuring the correct diagnosis. It was important to exclude other diagnoses for abdominal pain, such as cholecystitis, appendicitis, peptic ulcer disease, and splenic or hepatic infarction, and other causes for AP, namely, the presence of gallstones which was not uncommon in patients with very high BMI, such as ours. However, we cannot completely exclude this etiology. For excluding that, it would be advisable to make a magnetic resonance cholangiopancreatography (MRCP) to study the bile and pancreatic ducts and to exclude the presence of small stones. Although none of the patients had a MRCP to exclude lithiasis, the abdominal CT did not show lithiasis evidence and there was no analytical evidence of cholestasis. The suggestive history of higher alcohol consumption in one of our patients and the presence of SHTG in all of them appeared to be the cause of AP.

Normal TG metabolism maintains safe levels lower than 150 $\mathrm{mg} / \mathrm{dl}(12,13)$. Excessive ingestion, excessive synthesis, or ineffective TG clearance leads to HTG. Therefore, the causes of HTG may be categorized into primary disorders (genetically based) such as lipoprotein lipase (LPL) and apolipoprotein C-II deficiency, which are usually present in childhood as chylomicronemia syndromes, and secondary disorders such as obesity, untreated diabetes mellitus, alcoholism, pregnancy, drugs associated (estrogens, glucocorticoids, and thiazide diuretics) or lithiasis. Abnormalities of insulin responsiveness are present in some of these cases $(3,14)$. The association of SHTG and AP was first postulated by Speck in 1865 (15). SHTG is reported to account for up to $10 \%$ of all AP episodes (16). It is generally believed that TG levels of $>1000 \mathrm{mg} / \mathrm{dl}$ are a greater risk factor for AP (3). This threshold is arbitrary and the level above which AP might occur is actually unknown (4).

The exact pathophysiology still remains unclear. Several mechanisms have been proposed, including the genetic predisposition. The sheer size of TG can impede pancreatic blood flow and produce local acinar ischemia. The disturbance of the acinar structure liberates pancreatic lipase; this enzyme degrades chylomicrons and there is a production of pro-inflammatory free fatty acids. This pro-inflammatory state plays a major role in the systemic responses to AP and can accelerate pancreatic damage, producing local tissue necrosis $(12,13)$. To remember, our patients have some risk factors to HTG - those with type 2 diabetes, obesity, and hypothyroidism have reduced LPL activity, although the mechanism for this is unclear; therefore, the TG clearance is reduced. Acute alcohol consumption has been shown to increase plasma TG through two mechanisms: acute inhibition of LPL and increased hepatic synthesis of Very-low-density lipoprotein (VLDL).

The aim of the treatment is to reduce endogenous and exogenous sources of TG and promote their clearance. Exogenous sources can be reduced by low-fat diets and fasting; endogenous sources can be reduced by lipid-lowering medications and limited alcohol intake; clearance can be promoted by insulin and heparin. Insulin activates LPL and heparin stimulates the release of endothelial LPL into the circulation, leading to an acceleration of chylomicron degradation. Lifestyles such as dietary changes, avoidance of alcohol, weight reduction, exercise, and control of concomitant endocrinopathy are imperative measures in the management of SHTG.

All of these measures take time, and in patients with SHTG with hemodynamic instability or lack of response to medical treatment, such as the cases reported here, there is an urgent need of lowering the TG levels in order to prevent a multiorgan failure. PF consists in an extracorporeal separation of blood components resulting in a filtered plasma product, and it has some clinical applications. In the setting of SHTG, the goal is to remove the TG component of the blood. But PF is a nonselective removal process; so it could remove also circulating pro-inflammatory molecule stopping the underlying inflammatory cascade. Its use for lowering TG levels was first reported in 1978 by Betteridge et al. (14). Nowadays, experience with PF in acute hypertriglycerideinduced pancreatitis is limited to case reports and case series, and there are no consensus guidelines on optimal therapy. Although rare, HTG is the cause of AP in pregnancy in 50\% of cases and, in these cases, there is a greater consensus regarding its use because the use of fibrates is not recommended.

Ewald et al. (15) showed that a single session of PF can lower TG by $70 \%$. Similarly, Yeh (16) reported that a single cycle of PF lowered TG by $65 \%$ and a second cycle by $80 \%$. However, these authors did not find any association between the number of sessions and survival rates $(4,17,18)$. In the cases reported here, one or two sessions were sufficient. Ramirez et al. (17) described 11 patients submitted to PFin eight patients, a single plasma exchange was sufficient to reduce $\mathrm{TG}<1000 \mathrm{mg} / \mathrm{dl}$. Only three patients died, all with the worst severity indexes and who experienced most delay before the procedure (19). The Clinical Practice Guidelines recommend daily $\mathrm{PF}$ for $1-3$ days depending on the patient course and TG level $(15,20)$. The goal is to achieve TG concentrations lower than $500-1000 \mathrm{mg} / \mathrm{dl}$. Heparin use as anticoagulant for the procedure may have advantages considering its ability to release LPL, enhancing TG reduction (19).

Data suggest that PF should be performed as early as possible in order to achieve best results (21). Our patients underwent $\mathrm{PF}$ in the first $48 \mathrm{~h}$ after admission-they showed not only a rapid reduction of TG but also a clinical improvement.

The ASFA (6) conducted a literature review to evaluate the rationale use of $\mathrm{PF}$ in hypertriglyceridemic pancreatitis. This review is consistent with the findings of Chen and 
Gubensek (19) and reported the absence of randomized controlled trials about the effectiveness of the technique $(9,22)$. However, there has been an increasing number of successful cases described. It is also unclear whether PF increases hospital stay and/or mortality (22-24). Risks associated with PF are low but they exist. The risk of infection may be better weighed; PF does not selectively remove lipoproteins and TG; coagulation factors and immunoglobulins are also removed. Selective whole blood lipoprotein aphaeresis is feasible in some centers without the undesired consequences of $\mathrm{PF}$, but it causes only a moderate reduction in TG levels (18).

Bleeding and catheter-associated complications may occur. When $5 \%$ albumin is used, there is a progressive loss of coagulation factors. Thus, the patient's bleeding risk increases, especially as the initial treatment requires intensive anticoagulation to avoid system clotting. Hypersensitivity reactions to fresh frozen plasma if used as a replacement fluid have been reported (16). There is no data recommending one replacement fluid over another. Most studies have used 5\% albumin, while some have used fresh frozen plasma which contains LPL; this could enhance TG removal $(25,26)$.

We have to consider that PF is an expensive technique that is not available in all centers. Protocols should be created for standardized clinical practice. We suggest that in AP due to SHTG, PF should be performed concomitantly with medical therapy in the first $48 \mathrm{~h}$ after admission of patients with one or more of the following severity criteria: Ranson score on admission or in the first $48 \mathrm{~h}$ greater than or equal to 3 ; APACHE II score greater than 8 , at any time; presence of one or more organ failures; and presence of one or more local complications. We suggest the use of 5\% albumin replacement solution or fresh frozen plasma if there are hemorrhagic complications, coagulation abnormalities, or severe thrombocytopenia. PF should be performed until TG is less than $500 \mathrm{mg} / \mathrm{dl}$.

In conclusion, PF seems to be a relatively safe technique that should be considered as a complementary treatment to medical therapeutic in severe cases of acute hypertriglyceridemic pancreatitis, preferably in the early stages of the disease. A strictly pharmacologic treatment may be necessary for the prevention of SHTG that leads to AP, but lifestyle changes are also imperative.

Ideally, multicenter prospective studies should be conducted in order to provide a sufficient sample size in which the patients could be randomized to receive either conventional treatment or PF. However, it poses a big problem to the clinicians - to give or not to give a treatment that quickly removes the aggression trigger which may affect the patient recovery and even survival. It would be important to establish some guidelines for PF use, namely, the number of cycles that are necessary and the timing in which the process should be initiated. Maybe in the future, the process will be selective with using membrane-coated antibodies at low costs. Although rare, the complications of PF may contribute to morbidity or even mortality, especially in the hemodynamically compromised patients. We do not know whether there is an improvement in mortality. However, we should consider this technique if it is available in severe cases wherein the medical therapy is not fast enough to prevent the inflammatory cascade leading to multiorgan dysfunction.

Therefore, it is important to report all of similar cases to ascertain the efficacy, timing, and cost-benefit ratio of PF in the setting of AP due to SHTG. Maybe in the future, it can be used as a first-line therapy in selected cases as a bridge to medical treatment.

\section{Conflicts of interest}

The authors declare no conflicts of interest with respect to research, authorship, and/or publication of this article.

\section{References}

1. Cappell M. Acute pancreatitis: Etiology, clinical presentation, diagnosis, and therapy. Med Clin North Am. 2008;92(4):889-923. http://dx.doi.org/10.1016/j.mcna.2008.04.013

2. Nasa P, Alexander G, Kulkarni A, Juneja D, Sehra S, Agarwal $\mathrm{R}$, et al. Early plasmapheresis in patients with severe hypertriglyceridemia induced acute pancreatitis. Indian J Crit Care Med. 2015;19(8):487. http://dx.doi.org/10.4103/0972-5229.162472

3. Anderson F, Thomson S, Clarke D, Buccimazza I. Dyslipidaemic pancreatitis clinical assessment and analysis of disease severity and outcomes. Pancreatology. 2009;9(3):252-7. http://dx.doi.org/ $10.1159 / 000212091$

4. Ewald N, Kloer H. Treatment options for severe hypertriglyceridemia (SHTG): The role of apheresis. Clin Res Cardiol Suppl. 2012;7(Suppl 1):31-5. http://dx.doi.org/10.1007/s11789-012-0042-x

5. Eren G, Hergünsel O, Altun D, Cukurova Z, Yasar L. An alternative treatment in hypertriglyceridemia-induced acute pancreatitis in pregnancy: Plasmapheresis. J Anaesthesiol Clin Pharmacol. 2012;28(2):252. http://dx.doi.org/10.4103/0970-9185.94913

6. Winters J. American society for apheresis guidelines on the use of apheresis in clinical practice: Practical, concise, evidence-based recommendations for the apheresis practitioner. J Clin Apher. 2014;29(4):191-3. http://dx.doi.org/10.1002/jca.21334

7. Faydhi A, Mohidin S, Al Eidarous S. Use of plasmapheresis in acute pancreatitis secondary to hypertriglyceridemia. Univers J Med Sci. 2015;3(1):28-32. http://doi.org/10.13189/ujmsj.2015. 030104

8. Banks P, Freeman M. Practice guidelines in acute pancreatitis. Am J Gastroenterol. 2006;101(10):2379-400. http://dx.doi.org/ 10.1111/j.1572-0241.2006.00856.x

9. Tampieri A, Cenni P, Morselli C, Lenzi T. Plasmapheresis in hypertriglyceridemia-related pancreatitis: A case report. Emerg Care J. 2012;8(2):9. http://dx.doi.org/10.4081/ecj.2012.2.9

10. AbouRjaili G, Shtaynberg N, Wetz R, Costantino T, Abela G. Current concepts in triglyceride metabolism, pathophysiology, and treatment. Metabolism. 2010;59(8):1210-20. http://dx.doi. org/10.1016/j.metabol.2009.11.014

11. Collis L, Chambers D, Carr B. Hypertriglyceridaemia-induced acute pancreatitis: Is plasmapheresis really indicated? $3 \mathrm{C} 00$. J Intensive Care Soc. 2014;15(1):66-9. http://dx.doi.org/10. 1177/175114371401500115

12. Chang C-C, Hsieh YY, Tsai HD, Yang TC, Yeh LS, Hsu TY. Acute pancreatitis in pregnancy. Chin Med J. 1998;61(2):85-92. 
13. Sarwar N, Danesh J, Eiriksdottir G, Sigurdsson G, Wareham N, Bingham S, et al. Triglycerides and the risk of coronary heart disease: 10158 incident cases among 262525 participants in 29 western prospective studies. Circulation. 2007;115(4):450-8. http://dx.doi.org/10.1161/CIRCULATIONAHA.106.637793

14. Betteridge D, Bakowski M, Taylor K, Reckless J, De Silva S, Galton D. Treatment of severe diabetic hypertriglyceridemia by plasma exchange. Lancet. 1978;311(8078):1368. http://dx.doi. org/10.1016/S0140-6736(78)92450-9

15. Ewald N, Kloer H. Severe hypertriglyceridemia: An indication for apheresis? Atheroscler Suppl. 2009;10(5):49-52. http://dx. doi.org/10.1016/S1567-5688(09)71810-0

16. Yeh J, Chen J, Chiu H. Plasmapheresis for hyperlipidemic pancreatitis. J Clin Apher. 2003;18(4):181-5. http://dx.doi.org/10. 1002/jca. 10063

17. Ramírez-Bueno A, Salazar-Ramírez C, Cota-Delgado F, de la Torre-Prados M, Valdivielso P. Plasmapheresis as treatment for hyperlipidemic pancreatitis. Eur J Intern Med. 2014;25(2): 160-3. http://dx.doi.org/10.1016/j.ejim.2013.08.701

18. Rehmanjan M. Is plasmapheresis the optimal treatment option for acute pancreatitis secondary to hypertriglyceridemia? A systematic review. Discover Oppor Gen Pract. 2013;19-22.

19. Gubensek J, Buturovic-Ponikvar J, Romozi K, Ponikvar R. Factors affecting outcome in acute hypertriglyceridemic pancreatitis treated with plasma exchange: An observational cohort study. PLoS One. 2014;9(7):e102748. http://dx.doi.org/10.1371/ journal.pone. 0102748
20. Syed H, Bilusic M, Rhondla C, Tavaria A. Plasmapheresis in the treatment of hypertriglyceridemia-induced pancreatitis: A community hospital's experience. J Clin Apher. 2010;25(4):229-34. http://dx.doi.org/10.1002/jca.20232

21. Yadav D, Pitchumoni C. Issues in hyperlipidemic pancreatitis. J Clin Gastroenterol. 2003;36(1):54-62. http://dx.doi.org/10. 1097/00004836-200301000-00016

22. Maher $\mathrm{N}$, Ramaswamykanive $\mathrm{H}$. Use of plasmapheresis in managing the diagnostic dilemma of symptomatic hypertriglyceridemia. Case Rep Gastrointest Med. 2012;2012:1-3. http:// dx.doi.org/10.1155/2012/501373

23. Stefanutti C, Julius U. Treatment of primary hypertriglyceridemia states - General approach and the role of extracorporeal methods. Atheroscler Suppl. 2015;18:85-94. http://dx.doi.org/10.1016/j. atherosclerosissup.2015.02.017

24. Stefanutti C, Labbadia G, Morozzi C. Severe hypertriglyceridemiarelated acute pancreatitis. Ther Apher Dial. 2013;17(2):130-7. http://dx.doi.org/10.1111/1744-9987.12008

25. Zeitler H, Balta Z, Klein B, Strassburg C. Extracorporeal treatment in severe hypertriglyceridemia-induced pancreatitis. Ther Apher Dial. 2015;19(4):405-10. http://dx.doi.org/10.1111/17449987.12286

26. Schwartz J, Padmanabhan A, Aqui N, Balogun R, ConnellySmith L, Delaney M, et al. Guidelines on the use of therapeutic apheresis in clinical practice-evidence-based approach from the Writing Committee of the American Society for Apheresis: The seventh special issue. J Clin Apher. 2016;31(3):149-338. http:// dx.doi.org/10.1002/jca.21470 